\title{
Filter Convergence and Fuzzy Topology
}

\author{
Kyung Chan Min ${ }^{1}$, Yoon Jin Lee ${ }^{2}$ and Jae Deuk Myung ${ }^{3}$ \\ 1 Department of Mathematics, Yonsei University, Seoul 120-749, Korea \\ 2 Department of Applied Mathematics, Hankyong National University, Anseong, 456-749, Korea \\ 3 Department of General Studies, Korea Aerospace University, Goyang, 412-791, Korea
}

\begin{abstract}
After introducing many different types of prefilter convergence, we introduce an universal method to define various notions of compactness using cluster point and convergence of a prefilter and to prove the Tychonoff theorem using characterizations of ultra(maximal) prefilters.
\end{abstract}

Key Words : prefilter convergence, universal method, Tychonoff theorem, ultra prefilter, good extension

\section{Introduction}

In general topology the notion of convergence is one of main concepts which play essential role in theoretical development and its applications. In fact the fundamental notions, limit and continuity in topology are described naturally in terms of convergence. Particularly, filter convergence and net convergence are very useful notions to deal with compactness in terms of cluster points and limit points.

In fuzzy topology also the notion of convergence plays an important role to deal with limit and continuity. Moreover it is interesting to know that there exist various notions of convergence in a fuzzy topology $[1,3,4,5,8,9,14,18]$. On the other hand there exist various notions of compactness in a fuzzy topology using open sets, prefilters, fuzzy nets and functors between fuzzy topological spaces and topological spaces[2,6,7,10,11,15,17]. This means that we can discuss various types of compactness in fuzzy topology in terms of convergence.

In this chapter, first we introduce many different types of prefilter convergence. After finding an universal method to define the notions of compactness using cluster points and convergence of a prefilter, we prove the Tychonoff theorem using characterizations of ultra(maximal) prefilters. In [2], the Tychonoff theorems for $\alpha$-compactness and strong compactness, respectively, were proved using the Alexander Subbase Theorem. It is interesting to note that our approach provides a simple proof for the Tychonoff theorems as good extensions of compactness in a topological space. Various kinds of examples in a fuzzy topological space will be followed.

\section{Filter Convergence}

In a fuzzy topological space, there exist many different types of prefilter convergence depending on a notion of neighborhood of a point or a fuzzy point. First we introduce various notions of neighborhood in a fuzzy topological space.

\section{Neighbourhood (1) [18]}

Let $X$ be a fuzzy topological space and $x \in X$. A fuzzy set $V$ in $X$ is a neighborhood of $x$ if there exists an open set $U$ in $X$ such that $U \subseteq V$ and $U(x)=V(x)>0$.

\section{Neighbourhood (2) [1]}

A fuzzy set $V$ in $X$ is a neighborhood of a fuzzy point $p=(x, \alpha)(0<\alpha \leq 1)$ if there exists an open set $U$ in $X$ such that $p \subseteq U \subseteq V$.

\section{Neighbourhood (3) [16]}

A fuzzy set $V$ in $X$ is a neighborhood of a fuzzy point $p=(x, \alpha)(0<\alpha<1)$ if there exists an open set $U$ in $X$ such that $p \in U \subseteq V(p \in U$ means $\alpha<U(x))$.

\section{Neighbourhood (4) [14]}

A fuzzy set $V$ in $X$ is a neighborhood (Qneighborhood) of a fuzzy point $p=(x, \alpha)(0<\alpha \leq 1)$ if there exists an open set $U$ in $X$ such that $p q U \subseteq V(p q U$ means $U(x)+\alpha>1)$.

Remark. Wang [17] defined another notion of neighborhood in terms of closed set as follows : A closed set $C$ in $X$ is a neighborhood ( $R$-neighborhood) of a fuzzy point $p=(x, \alpha)(0<\alpha \leq 1)$ if $p \nsubseteq C$. In fact, it is easy to see that the notions Q-neighborhood and R-neighborhood are equivalent. 
Now we introduce various types of prefilter convergence.

Let $x \in X, \alpha \in(0,1]$ or $(0,1)$ and $p=(x, \alpha)$, a fuzzy point $X$. We denote $\mathcal{N}^{i}(p)=$ the collection of all neighborhood $(i)$ s of $p$ for each $i=1,2,3,4$. For $i=1$, we mean $p=(x, 1)$.

Convergence (I), (II), (III), (IV) : We say that $p$ is a cluster point of a prefilter $\mathcal{F}$ on $X$ if $U \cap F \neq \emptyset$ for all $U \in \mathcal{N}(p)$ and $F \in \mathcal{F}$. It is said that a prefilter $\mathcal{F}$ on $X$ converges to $p$ and write $\mathcal{F} \rightarrow p$, if $\mathcal{N}(p) \subseteq \mathcal{F}$. Clearly, if $\mathcal{F} \rightarrow p$ in $X$, then $p$ is a cluster point of $\mathcal{F}$.

Convergence (V) : We say that $p$ is a cluster point of a prefilter $\mathcal{F}$ on $X$ if $U q F$ for all $U \in \mathcal{N}(p)$ and $F \in \mathcal{F}$ ( $U q F$ means that $U(x)+F(x)>1$ for some $x \in X$ ). We say that a prefilter $\mathcal{F}$ on $X$ converges to $p$ and write $\mathcal{F} \rightarrow p$, if for any neighborhood $U$ of $p$, there exists $F \in \mathcal{F}$ such that for every $F^{\prime} \subseteq F, U(x)+F^{\prime}(x)>1$ for every $x \in S u p p F^{\prime}$. We note that if $\mathcal{F} \rightarrow p$ in $X$, then $p$ is a cluster point of $\mathcal{F}$.

Convergence (VI) : A prefilter $\mathcal{F}$ on $X$ is called a $\alpha$ prefilter if $\mathcal{F}$ does not contain the constant function $c_{\alpha}$, $(0 \leq \alpha<1)$. We note that a prefilter $\mathcal{F}$ on $X$ is an $\alpha$ prefilter iff $F^{-1}(\alpha, 1] \neq \emptyset$ for every $F \in \mathcal{F}$. We denote by $F(X), P(X)$ the collection of filters, respectively prefilters on $X$. We denote by $P^{\alpha}(X)$ the collection of all $\alpha$-filters on $X$. We define a correspondence $\iota_{\alpha}: P^{\alpha}(X) \rightarrow F(X)$ by $\iota_{\alpha}(\mathcal{F})=\left\{U^{-1}(\alpha, 1]: U \in \mathcal{F}\right\}$. Let $(X, \delta)$ be a fuzzy topological space. For each $\alpha \in[0,1)$, we consider the topology $\iota_{\alpha}(\delta)=\left\{U^{-1}(\alpha, 1]: U \in \delta\right\}$ on $X$. Let $(X, \tau)$ be a topological space. For each $\alpha \in[0,1)$, we consider the fuzzy topology $\omega_{\alpha}(\tau)=\left\{U \in I^{X}: U^{-1}(\alpha, 1] \in \tau\right\}$ on $X$. We say that $p=(x, \alpha)$ is a $\alpha$-cluster point of a prefilter $\mathcal{F}$ on $X$ if $\mathcal{F}$ is a $\alpha$-prefilter and $x$ is a cluster point of the filter $\iota_{\alpha}(\mathcal{F})$ in $\left(X, \iota_{\alpha}(\delta)\right)$. We say that $\mathcal{F} \alpha$-converges to $p=(x, \alpha)$, denote by $\mathcal{F} \stackrel{\alpha}{\rightarrow} p$, if $\mathcal{F}$ is a $\alpha$-prefilter and the filter $\iota_{\alpha}(\mathcal{F})$ converges to $x$ in $\left(X, \iota_{\alpha}(\delta)\right)$.

Theorem 2.1. Let $\left\{f_{i}: X \rightarrow X_{i}\right\}_{\Lambda}$ be a family of fuzzy continuous maps. Assume that $X$ has an initial fuzzy topology with respect to $\left\{f_{i}\right\}_{\Lambda}$. For any given convergence among Convergences (I), (II), (III), (IV), (V) and (VI) $\mathcal{F} \rightarrow p$ in $X$ if and only if $f_{i}(\mathcal{F}) \rightarrow f_{i}(p)$ in $X_{i}$ for any $i \in \Lambda$.

Proof. It is routine. For convergence (V), we use the fact $p q \cap_{i=1}^{n} A_{i}$ if and only if $p q A_{i}$ for all $i=1, \cdots, n$. For convergence $(\mathrm{VI})$, we use the fact that the correspondence $\iota_{\alpha}$ preserves initial families. (Cf. Theorem 1.5 in [6]).

\section{Ultra Compact Spaces}

In this section we introduce a number of conditions for an universal scheme to define a notion of ultrafilter compactness and to obtain the Tychonoff theorem.

Let $X$ be a fuzzy topological space. Let $\alpha \in(0,1]$ or $(0,1)$. Let $S_{X} \subseteq P(X)$. Let $C_{X} \subseteq S_{X} \times P_{X}$ and $L_{X} \subseteq S_{X} \times P_{X}$, where $P_{X}=\{(x, \alpha): x \in X\}$.

(P1) $(\mathcal{F}, p) \in C_{X}$ if and only if there exists $\mathcal{G} \in S_{X}$ such that $\mathcal{G} \supseteq \mathcal{F}$ and $(\mathcal{G}, p) \in L_{X}$

(P2) for every element $\mathcal{F} \in S_{X}$, there exists a maximal element in $S_{X}$ containing $\mathcal{F}$ with respect to inclusion.

(P3) if $f: X \rightarrow Y$ is a map and $\mathcal{F}$ is a maximal element in $S_{X}$, then $f(\mathcal{F})$ is a maximal element in $S_{Y}$.

(P4) $(\mathcal{F}, p) \in L_{\prod_{i} X_{i}}$ if and only if $\left(\pi_{i}(\mathcal{F}), \pi_{i}(p)\right) \in L_{X_{i}}$ for each $i$.

From now on, we assume that every fuzzy topological space $X$ is equipped with a triples $\left(S_{X}, C_{X}, L_{X}\right)$ satisfying the conditions (P1), (P2), (P3) and (P4).

Definition 3.1. A space $X$ is called an ultrafilter $\alpha$ compact space if for every maximal element $\mathcal{U}$ in $S_{X}$, there exists $p \in P_{X}$ such that $(\mathcal{U}, p) \in L_{X}$.

Theorem 3.2. A space $X$ is ultrafilter $\alpha$-compact if and only if for every element $\mathcal{F}$ in $S_{X}$ there exists $p \in P_{X}$ such that $(\mathcal{F}, p) \in C_{X}$.

Proof. $(\Rightarrow)$ For any element $\mathcal{F}$ in $S_{X}$, there exists a maximal element $\mathcal{U}$ in $S_{X}$ containing $\mathcal{F}$ with respect to inclusion by (P2). Since there exists $p \in P_{X}$ such that $(\mathcal{U}, p) \in L_{X}$, and $\mathcal{F} \subseteq \mathcal{U},(\mathcal{F}, p) \in C_{X}$ by $(\mathbf{P 1})$.

$(\Leftarrow)$ Let $\mathcal{U}$ be a maxiaml element in $S_{X}$. Then there exists $p \in P_{X}$ such that $(\mathcal{U}, p) \in C_{X}$. Hence $(\mathcal{U}, p) \in L_{X}$ by (P1).

Theorem 3.3. (Tychonoff) Let $\left\{X_{i}\right\}_{\Lambda}$ be a family of spaces. Then the product space $\prod_{i} X_{i}$ is ultrafilter $\alpha$ compact if and only if so is $X_{i}$ for each $i \in \Lambda$.

Proof. $(\Rightarrow)$ For each $i \in \Lambda$, let $\mathcal{A}$ be a maximal element in $S_{X_{i}}$ and $\mathcal{F}$ the prefilter generated by $\left\{\pi_{i}^{-1}(A): A \in\right.$ $\mathcal{A}$ \}. Let $\mathcal{U}$ be a maximal element in $S_{\prod_{i} X_{i}}$ containing $\mathcal{F}$ by (P2). Since $\prod_{i} X_{i}$ is ultrafilter $\alpha$-compact, there exists $p \in P \prod_{\prod_{i} X_{i}}$ such that $(\mathcal{U}, p) \in L_{\prod_{i} X_{i}}$ and hence, $\left(\pi_{i}(\mathcal{U}), \pi_{i}(p)\right) \in L_{X_{i}}$ by $(\mathbf{P 4})$. Note that $\mathcal{A}=\pi_{i}(\mathcal{F})=$ $\pi_{i}(\mathcal{U})$.

$(\Leftarrow)$ Let $\mathcal{U}$ be a maximal element in $S_{\prod_{i} X_{i}}$. Then $\pi_{i}(\mathcal{U})$ is a maximal element in $S_{X_{i}}$ for each $i$, by (P3). Let $\left(\pi_{i}(\mathcal{U}), \pi_{i}(p)\right) \in L_{X_{i}}$ for each $i$. Let $p=\left(\left(x_{i}\right), \alpha\right)$, where $p_{i}=\left(x_{i}, \alpha\right)$. Then by (P4) $(\mathcal{U}, p) \in L \prod_{i} X_{i}$.

We introduce one more condition: 
(P5) if $f: X \rightarrow Y$ is fuzzy continuous, then whenever $(\mathcal{F}, p) \in L_{X},(f(\mathcal{F}), f(p)) \in L_{Y}$.

Theorem 3.4. Let $f: X \rightarrow Y$ be a fuzzy continuous onto map. Assume that $X$ and $Y$ satisfy (P3) and (P5). If $X$ is ultra $\alpha$-compact, then so is $Y$.

Proof. It is obvious by a smilar method in the proof of Theorem 3.3.

Assume that every fuzzy topological space $X$ is equipped with a triple $\left(S_{X}, C_{X}, L_{X}\right)$ satisfies the conditions (P1), (P2), (P3), (P4) and (P5).

Definition 3.5. A space $X$ is called an ultrafilter compact space if it is ultrafilter $\alpha$-compact for each $\alpha$.

By Theorem 3.4, we have the following result.

\section{Theorem 3.6.}

1. Let $f: X \rightarrow Y$ be a fuzzy continuous onto map. If $X$ is ultrafilter compact, then so is $Y$.

2. The Tyconoff theorem holds for ultrafilter compact spaces.

\section{Ultrafilters}

The characterization of an ultrafilter on a set $X$ in terms of a subset of $X$ is important to calculate in many problems using ultrafilters. In this section we introduce characterizations of ultra prefilter depending on types of prefilter, respectively, and use them to obtain results in Section 3. First, we introduce a characterization of a prefilter. (See [12] for details)

Definition 4.1. A prefilter $\mathcal{F}$ on a set $X$ is an ultrafilter if there is no strictly finer prefilter than $\mathcal{F}$.

Definition 4.2. Let $\mathcal{F}$ be a prefilter on $X$. We say that a subset $Y$ of $X$ is included in $\mathcal{F} /$ if every fuzzy set in $X$ with support $Y$ is an element of $\mathcal{F}$.

Theorem 4.3. Let $\mathcal{F}$ be a prefilter on $X$. Then the following are equivalent,

1. $\mathcal{F}$ is an ultrafilter.

2. Let $A \in I^{F}$. If $A \notin \mathcal{F}$, then there is some $F \in \mathcal{F}$ such that $A \cap F=\emptyset$.

3. Let $T \subseteq X$. Then either $T$ or $T^{C}$ is included in $\mathcal{F}$.
Theorem 4.4. Let $f: X \rightarrow Y$ be a map. If $\mathcal{F}$ is an ultrafilter on $X$, then $f(\mathcal{F})$ is an ultrafilter on $Y$.

Now we introduce a characterization of an ultra $\bar{\alpha}$-filter. (See [12] for details)

Definition 4.5. A prefilter $\mathcal{F}$ in $X$ is called an $\alpha$-filter $(0<\alpha \leq 1)$ if $\inf _{F \in \mathcal{F}} h g t(F)=\alpha$, where $\operatorname{hgt}(F)=$ $\sup _{x \in X} \mu_{F}(x)$, the height of $F$. An $\alpha$-filter $\mathcal{F}$ on $X$ is called an $\bar{\alpha}$-filter if $F_{\alpha}=F^{-1}([\alpha, 1]) \neq \emptyset$ for every $F \in \mathcal{F}$, where $F_{\alpha}$ is the $\alpha$-cut of $F$.

Definition 4.6. An $\bar{\alpha}$-filter $\mathcal{F}$ on $X$ is called an ultra $\bar{\alpha}$ filter if there is no strictly finer $\bar{\alpha}$-filter than $\mathcal{F}$.

Definition 4.7. Let $\mathcal{F}$ be a prefilter on $X$ and $S \subseteq X$. S is called $\alpha$-included in $\mathcal{F}$ if every fuzzy set $A$ in $X$ with $\alpha$-cut $S$ is contained in $\mathcal{F}$.

Theorem 4.8. Let $X$ be a set and $\mathcal{F}$ an $\bar{\alpha}$-filter on $X$. Then the following are equivalent.

1. $\mathcal{F}$ is an ultra $\bar{\alpha}$-filter.

2. If $A \in I^{X}$ and $A \notin \mathcal{F}$ then $A \cap F<\alpha$ for some $F \in \mathcal{F}$.

3. If $S \subseteq X$ then either $S$ or $S^{C}$ is $\alpha$-included in $\mathcal{F}$.

Theorem 4.9. Let $X$ and $Y$ be sets and $f: X \rightarrow Y$ a map. If $\mathcal{F}$ is an $\alpha$-filter (resp. $\bar{\alpha}$-filter, ultra $\bar{\alpha}$-filter) on $X$, then $f(\mathcal{F})$ is an $\alpha$-filter (resp. $\bar{\alpha}$-filter, ultra $\bar{\alpha}$-filter) on $Y$.

Remark. G.Wang [17] defined a notion of N-compactness in terms of $\alpha$-filter: A fuzzy subset in $X$ is $\mathrm{N}$-compact iff each $\alpha$-filter $\mathcal{F}(\alpha \in(0,1])$ has a cluster point $(x, \alpha) \in A$, whenever $A \in \mathcal{F}$. He proved the Tychonoff theorem for $\mathrm{N}$-compactness using $\alpha$-net. We note that if we can have a characterization of an ultra $\alpha$-filter similar to Theorem 4.8 to obtain Theorem 4.9, the proof of the Tychonoff theorem for $\mathrm{N}$-compactness should be much simpler than the Wang's proof in [17].

Now, we introduce a characterization of an ultra $t$ prefilter

Definition 4.10. [13] A $\alpha$-prefilter $\mathcal{F}$ on $X$ is called an $u l$ tra $\alpha$-prefilter (=maximal $\alpha$-prefilter) if there is no strictly finer $\alpha$-prefilter than $\mathcal{F}$. 
Definition 4.11. [13] Let $\mathcal{F}$ be $\alpha$-prefilter on $X$. We say that a subset $\mathrm{T}$ of $X$ is $\alpha$-included in $\mathcal{F}$ if every fuzzy set $A$ in $X$ with $A^{-1}(\alpha, 1]=T$ is an element of $\mathcal{F}$.

Theorem 4.12. [13] Let $\mathcal{F}$ be a $\alpha$-prefilter. Then the following statements are equivalent.

1. $\mathcal{F}$ is an ultra $\alpha$-prefilter.

2. For every $A \in I^{X}$ such that $A^{-1}(\alpha, 1] \neq \emptyset$, if $A \notin$ $\mathcal{F}$, then there exists $F \in \mathcal{F}$ such that $A \cap F \subseteq c_{\alpha}$.

3. For every $T \subseteq X$, either $T$ or $T^{C}$ is $\alpha$-included in $\mathcal{F}$.

Theorem 4.13. Let $f: X \rightarrow Y$ be a map. If $\mathcal{F}$ is a $\alpha$-prefilter (resp. ultra $\alpha$-prefilter) on $X$, then $f(\mathcal{F})$ is a $\alpha$-prefilter (resp. ultra $\alpha$-prefilter) on $Y$.

Proof. Suppose $f(\mathcal{F})$ is not an $\alpha$-prefilter, i.e. $c_{\alpha} \in$ $f(\mathcal{F})$. Then $f(\mathcal{F}) \subseteq c_{\alpha}$ for some $F \in \mathcal{F}$ and hence $F \subseteq f^{-1}\left(c_{\alpha}\right)=c_{\alpha}$. Therefore we have $c_{\alpha} \in \mathcal{F}$, which is a contradiction. Let $\mathcal{F}$ be an ultra $\alpha$-prefilter on $X$. Let $T \subseteq Y$ and $S=f^{-1}(T) \subseteq X$. Then either $S$ or $S^{C}$ is $\alpha$-included in $\mathcal{F}$ by Thoerem 4.12. Suppose $S$ is $\alpha$-included in $\mathcal{F}$. Let $A$ be a fuzzy set in $Y$ with $A^{-1}(\alpha, 1]=T$. Put $B=f^{-1}(A)$. Then $B^{-1}(\alpha, 1]=$ $f^{-1}\left(A^{-1}(\alpha, 1]\right)=f^{-1}(T)=S$ and hence $B \in \mathcal{F}$. Now $f(B)=f\left(f^{-1}(A)\right) \subseteq A$ and hence $A \in f(\mathcal{F})$. Suppose $S^{C}$ is $\alpha$-included in $\mathcal{F}$. Then by a similar argument we can show that $T^{C}$ is $\alpha$-included in $f(\mathcal{F})$. Therefore $f(\mathcal{F})$ is an ultra $\alpha$-prefilter on $Y$ by Theorem 4.12 .

\section{Applications}

Using the universal scheme for ultrafilter compactness, we introduce various types of an ultrafilter compact spaces depending on prefilter Convergences (I), (II), (III), (IV), (V) and (VI), respectively definded in Section 2. We show that each notion of ultrafilter compactness satisfies the conditions (P1), (P2), (P3), (P4) and (P5). This means each prefilter convergence provides Theorems 3.2, 3.3, 3.4, 3.5 and 3.6.

\section{A. Convergence (I)}

For a fuzzy topological space $X$, let $S_{X}=P(X), C_{X}=$ $\left\{(\mathcal{F}, p) \in S_{X}: p\right.$ is a cluster point of $\mathcal{F}$ in $\left.X\right\}$ and $L_{X}=\left\{(\mathcal{F}, p) \in S_{X}: \mathcal{F} \rightarrow p\right.$ in $\left.X\right\}$, where $p \in P_{X}=$ $\{(x, 1): x \in X\}$. It is known [4] that (P1) and (P3) hold for the triple $\left(S_{X}, C_{X}, L_{X}\right)$. By Zorn's Lemma, it is easy to check that the condition (P2) holds. By Proposition 2.1, the condition (P4) holds. Clearly the condition (P5) holds.

B. Convergence (II), (III), (IV)
For a fuzzy topological space $X$, take $\alpha \in(0,1]$ or $(0,1)$ and let $S_{X}=P(X), C_{X}=\left\{(\mathcal{F}, p) \in S_{X}: p\right.$ is a cluster point of $\mathcal{F}$ in $X\}$ and $L_{X}=\left\{(\mathcal{F}, p) \in S_{X}: \mathcal{F} \rightarrow p\right.$ in $X\}$, where $p \in P_{X}=\{(x, \alpha): x \in X\}$. It is known [11] that (P1) and (P3) hold for the triple $\left(S_{X}, C_{X}, L_{X}\right)$ for convergence (III). By similar methods in [12], we can show that (P1) and (P3) hold for convergence (II), (III), respectively. Moreover, it is routine to check that the conditions (P2), (P4) and (P5) hold for convergence (II), (III), (IV), respectively.

C. The Convergence (III) can give a different type of ultrafilter compactness as follows. A fuzzy point $p=(x, \alpha)$ is called an $\alpha$-cluster point of a prefilter $\mathcal{F}$ on $X$ if for every $U \in \mathcal{N}(p)$ and for every $F \in \mathcal{F},(U \cap F)_{\alpha} \neq \emptyset$. We note that if a prefilter $\mathcal{F}$ on $X$ has an $\alpha$-cluster point, then it is an $\bar{\alpha}$-filter. Let $\alpha \in(0,1)$ and $S_{X}=$ the collection of all $\bar{\alpha}$-filters on $X$. Let $C_{X}=\{(\mathcal{F}, p): p$ is an $\alpha$-cluster point of $\mathcal{F}\}$ and $L_{X}=\{(\mathcal{F}, p): \mathcal{F} \rightarrow p$ in $X\}$.

(P1) holds. Let $(\mathcal{F}, p) \in C_{X}$ and let $\mathcal{B}=\{U \cap F: U \in$ $\mathcal{N}(p), F \in \mathcal{F}\}$, which is a prefilter base on $X$. Then the prefilter $\mathcal{G}=[\mathcal{B}]$ is an $\bar{\alpha}$-filter and $\mathcal{G} \rightarrow x$ in $X$. It is easy to see that the converse is true. By routine process, we can show that (P2) holds. By Theorem 2.6 in [11], we have (P3). Clearly (P4) and (P5) hold by Theorem 2.1. Min and Kim [11] called an ultrafilter $\alpha$-compact space in this section as a stable fuzzy compact and showed the Tychonoff theorem.

Remark 5.1 Min and Kim [11] compared ultrafilter $\alpha$ compactness with other notions of compactness:

strong fuzzy compact $\Rightarrow$ ultrafilter compact $\Rightarrow$ fuzzy compact None of the arrows is revisible. Hence by Theorem 5.7 in [17] strong fuzzy compactness, ultrafilter compactness and fuzzy compactness are equivalent in a Hausdorff fuzzy topological space.

\section{Convergence (V)}

For a fuzzy topological space $X$, take $\alpha \in(0,1]$ and let $S_{X}=P(X), C_{X}=\left\{(\mathcal{F}, p) \in S_{X}: p\right.$ is a cluster point of $\mathcal{F}$ in $X\}$ and $L_{X}=\left\{(\mathcal{F}, p) \in S_{X}: \mathcal{F} \rightarrow p\right.$ in $\left.X\right\}$, where $p \in P_{X}=\{(x, \alpha): x \in X\}$. The condition (P1) follows form Theorem 13.2 [14]. The condition (P2) can be obtained by a usual method using Zorn's Lemma. The condition (P3) follows form Theorem 4.4. The conditions (P4) and (P5) follow from Theorem 3.7 and Proposition 3.5 [8], respectively.

\section{E. Convergence (VI)}

For $\alpha \in[0,1)$, let $S_{X}=$ the collection of all $\alpha$-prefilters on $X$. Let $C_{X}=\{(\mathcal{F}, p): p$ is a $\alpha$-cluster point of $\mathcal{F}$ in $X\}$ and $L_{X}=\{(\mathcal{F}, p): \mathcal{F} \stackrel{\alpha}{\rightarrow} p$ in $X\}$. (P1) holds by Proposition 5.2 in [13]. By routine work, we can show that (P2) holds. (P3) holds by Theorem 4.13. Moreover it is easy to check that (P4) and (P5) hold, since the correspondence $\iota_{\alpha}$ preserves initial sources. (Cf. Theorem 1.5 in [6]) 
Remark 5.2 De Prada Vincente and Macho Stadler [13] showed that a fuzzy topological space $(X, \delta)$ is ultrafilter $\alpha$-compact for Convergence (VI) iff it is strong fuzzy compact. In [2], the Tychonoff theorems for $\alpha$-compactness and strong compactness, respectively, were proved using the Alexander Subbase Theorem. Therefore our approach using ultrafilter provides a different and simple proof for the Tychonoff theorem.

\section{Good extensions}

In this section we show that various types of ultrafilter compactness depending on the notions of convergence of prefilter introduced in Section 5 are good extensions of compactness in a topological space.

Let $\mathcal{F}$ be an $\alpha$-prefilter on a set $X(0 \leq \alpha<1)$. Then we know that $\iota_{\alpha}(\mathcal{F})=\left\{F^{-1}(\alpha, 1]: F \in \mathcal{F}\right\}$ is a filter on $X$. We note that a 0 -prefilter means a prefilter. Let $\mathbf{F}$ be a filter on a set $X$. Then for $\alpha \in[0,1), \omega_{\alpha}(\mathbf{F})=\left\{F \in I^{X}\right.$ : $\left.F^{-1}(\alpha, 1] \in \mathbf{F}\right\}$ is an $\alpha$-prefilter on $X$.

Proposition 6.1. Let $\mathcal{F}, \mathbf{F}$ be an $\alpha$-prefilter, respectively a filter on $X$ and $\alpha \in[0,1)$. Then

1. $\iota_{\alpha} \circ \omega_{\alpha}(\mathbf{F})=\mathbf{F}$.

2. $\mathcal{F} \subseteq \omega_{\alpha} \circ \iota_{\alpha}(\mathcal{F})$ and hence $\mathcal{F}=\omega_{\alpha} \circ \iota_{\alpha}(\mathcal{F})$ for an ultrafilter $\mathcal{F}$.

Proof. It is straightforward by definitions.

Let $\mathcal{F}$ be an $\bar{\alpha}$-filter on a set $X(0<\alpha<1)$. Then $\iota_{\alpha}{ }^{*}(\mathcal{F})=\left\{F^{-1}[\alpha, 1]: F \in \mathcal{F}\right\}$ is a filter on $X$. Let $\mathbf{F}$ be a filter on a set $X(0<\alpha \leq 1)$. Then $\omega_{\alpha}{ }^{*}(\mathbf{F})=\{F \in$ $\left.I^{X}: F^{-1}[\alpha, 1] \in \mathbf{F}\right\}$ is an $\bar{\alpha}$-filter on $X$. By a routine work we have the following:

Proposition 6.2. Let $\mathcal{F}, \mathbf{F}$ be an $\alpha$-filter, respectively a filter on $X$ and $\alpha \in[0,1)$. Then

1. $\iota_{\alpha}^{*} \circ \omega_{\alpha}^{*}(\mathbf{F})=\mathbf{F}$.

2. $\mathcal{F} \subseteq \omega_{\alpha}^{*} \circ \iota_{\alpha}^{*}(\mathcal{F})$ and hence $\mathcal{F}=\omega_{\alpha}^{*} \circ \iota_{\alpha}^{*}(\mathcal{F})$ for an ultrafilter $\mathcal{F}$.

\section{Proposition 6.3.}

1. If $\mathcal{U}$ is an ultrafilter in $P(X)$, then $\iota_{\alpha}(\mathcal{U})$ and $\iota_{\alpha}^{*}(\mathcal{U})$ are ultrafilters in $F(X)$ for $\alpha \in[0,1)$.

2. If $\mathbf{A}$ is an ultrafilter in $F(X)$, then $\omega_{\alpha}(\mathbf{A})$ and $\omega_{\alpha}^{*}(\mathbf{A})$ are ultrafilters in $P(X)$ for $\alpha \in[0,1)$.
Proof. (1) Let $\iota_{\alpha}(\mathcal{U}) \subseteq \mathbf{A}$ for a filter $\mathbf{A}$ on $X$. Then $\mathcal{U} \subseteq \omega_{\alpha} \circ \iota_{\alpha}(\mathcal{U}) \subseteq \omega_{\alpha}(\mathbf{A})$, i.e. $\mathcal{U}=\omega_{\alpha}(\mathbf{A})$. Hence $\iota_{\alpha}(\mathcal{U})=\iota_{\alpha} \circ \omega_{\alpha}(\mathbf{A})=\mathbf{A}$.

(2) Suppose $\mathcal{G}$ is a prefilter on $X$ such that $\omega_{\alpha}(\mathbf{A}) \subset \mathcal{G}$. Then there exists $G \in \mathcal{G}$ such that $G \notin \omega_{\alpha}(\mathbf{A})$, i.e. $G^{-1}(\alpha, 1] \notin \mathbf{A}$. Hence $\mathbf{A}=\iota_{\alpha} \circ \omega_{\alpha}(\mathbf{A}) \subset \iota_{\alpha}(\mathcal{G})$, which is a contradiction. By a similar method we obtain the results related to $\iota_{\alpha}^{*}$ and $\omega_{\alpha}^{*}$.

Proposition 6.4. Let $\mathbf{F}$ be a filter on a topological space $(X, \tau)$. Let $\mathbf{F} \rightarrow x$ in $X$. Then

1. $\omega_{\alpha}(\mathbf{F})\left(\right.$ resp. $\left.\omega_{\alpha}^{*}(\mathbf{F})\right) \rightarrow(x, \alpha)$ in $(X, \omega(\tau))$ for each $\alpha \in[0,1]$ for Convergences (I), (II), (III), (IV) and (V). For Convergence (I), we mean $\alpha=1$.

2. $\omega_{\alpha}(\mathbf{F}) \rightarrow(x, \alpha)$ in $(X, \omega(\tau))$ for Convergence $(\mathrm{VI})$.

Proof. (1) First we show for Convergences (I) and (II). Let $U:(X, \tau) \rightarrow I$ be a lower semicontinuous map such that $U(X)>\alpha$. Then there exists $A \in \mathbf{F}$ such that $A \subseteq U^{-1}(\alpha, 1]$ which implies $U^{-1}(\alpha, 1] \in \mathbf{F}$. Hence $U \in \omega(\mathbf{F})$. Therefore $\omega_{\alpha}(\mathbf{F}) \rightarrow(x, \alpha)$ in $(X, \omega(\tau))$. For other Convergences, we show the results by a similar method.

(2) Since $\iota_{\alpha} \circ \omega_{\alpha}(\mathbf{F})=\mathbf{F}$ and $\iota_{\alpha}(\omega(\tau))=\tau$, the result follows.

By definitions, we have the following:

Proposition 6.5. Let $\mathcal{F}$ be an $\alpha$-prefilter on a fuzzy topological space $(X, \delta)$ and $\alpha \in[0,1)$. Let $\mathcal{F} \rightarrow(x, \alpha)$ in $X$. Then

1. $\iota_{0}(\mathcal{F}) \rightarrow x$ in $\left(X, \iota_{0}(\delta)\right)$ for Convergence $(\mathrm{I})$.

2. $\iota_{\alpha}^{*}(\mathcal{F}) \rightarrow x$ in $\left(X, \iota_{\beta}(\delta)\right)$ for Convergence (III) for each $\beta<\alpha$.

3. $\iota_{\alpha}(\mathcal{F}) \rightarrow x$ in $\left(X, \iota_{\alpha}(\delta)\right)$ for Convergence (VI).

Proposition 6.6. A topological space $(X, \tau)$ is compact iff a fuzzy topological space $(X, \omega(\tau))$ is ultrafilter compact with respect to the notions in Parts A, C and D in Section 5 , respectively.

Proof. $(\Rightarrow)$ For Parts $\mathrm{A}$ and $\mathrm{D}$, let $\mathcal{U}$ be an ultrafilter in $P(X)$. Then $\iota_{\alpha}(\mathcal{U})$ is an ultrafilter in $F(X)$ for $\alpha \in[0,1)$. Since $(X, \tau)$ is compact, $\iota_{\alpha}(\mathcal{U}) \rightarrow x$ in the topological space $X$ for some $x \in X$. Hence $\mathcal{U}=\omega_{\alpha} \circ \iota_{\alpha}(\mathcal{U}) \rightarrow$ $(x, \alpha)$ in $(X, \omega(\tau))$ for each $\alpha \in(0,1]$. The reasult for Part $\mathrm{C}$ follows by a similar argument.

$(\Leftarrow)$ For Parts A and D, let $\mathbf{A}$ be an ultrafilter in $F(X)$. Then $\omega_{\alpha}(\mathbf{A})$ is an ultrafilter in $P(X)$ for $\alpha \in[0,1)$. Since $\omega_{\alpha}(\mathbf{A})$ is an $\alpha$-prefilter on $X, \omega_{\alpha}(\mathbf{A}) \rightarrow(x, \alpha)$ in $(X, \omega(\tau))$ by Proposition 6.4. By definition, $\mathbf{A}=$ $\iota_{\alpha} \circ \omega_{\alpha}(\mathbf{A}) \rightarrow x$ in $(X, \tau)$. Hence the result follows. The result for Part $\mathrm{C}$ follows by a similar argument.

By the above Proposition 6.6, we have the following: 
Corollary 6.7. The ultrafilter $(\alpha-)$ compactness defined in Parts A, C and D, respectively, is a good extension of the compactness in a topological space.

Remark. The result for Part $\mathrm{C}$ can obtained also from $\mathrm{Re}-$ mark 5.1.

\section{References}

[1] C.De Mitri and E. Pascali, "Charaterization of fuzzy topologies from neighborhoods of fuzzy points", $J$. Math. Anal. Appl. vol. 93, pp. 1-14, 1985.

[2] T.E.Gantner, R.C.Steinlage and R.H.Warren, "Compactness in fuzzy topological spaces”, J. Math. Anal. and Appl. vol. 62, pp. 547-562, 1978.

[3] Mutlu Guloglu and Dogan Coker, "Convergence in I-fuzzy topological spaces", Fuzzy sets and Systems vol. 151, pp. 615-623, 2005.

[4] A.T.Katsaras, "Convergence of fuzzy filters in fuzzy topological spaces”, Bull. Math.de la Soi. Sci.Math. dela R.S. de Roumanie Tome vol. 27(75), pp. 131-137, 1983.

[5] C.Y.Kim, "Fuzzy topology and neighbourhood systems", preprint.

[6] R.Lowen, "Initial and final fuzzy topologies and the fuzzy Tchychonoff theorem", J. Math. Anal. and Appl. vol. 58, pp. 11.-21, 1977.

[7] R.Lowen, "A comparison of different compactness notions in fuzzy topological spaces", J. of Math. Anal. and Appl. vol. 64, pp. 446-454, 1978.

[8] R.Lowen, "Convergence in fuzzy toplogical spaces", Gen.Top. and its Appl. vol. 10, pp.147-160, 1979.

[9] K.C.Min, "Fuzzy limit spaces", Fuzzy Sets and Systems vol. 322, pp. 343-357, 1989.

[10] K.C.Min and S.J.Lee, "Solid fuzzy compactness spaces", Yonsei Univ. ,1988.
[11] K.C.Min and Y.B.Kim, "Stable fuzzy compactness", Fuzzy Logic and its Applications. pp. 315-323, 1995.

[12] M.A.de Prada Vincente and M.Saralegui Aranguren, "Fuzzy filters", J. of Math. Anal. and Appl. vol. 129, pp. 560-568, 1988.

[13] M.A.de Prada Vincente and M.Macho Stadler, "tprefilter theory", Fuzzy Sets and Systems vol. 38, pp. 115-124, 1970.

[14] P-M.Pu and Y-M.Liu, "Fuzzy topology I. Neighborhood structure of a fuzzy point and Moore-Smith convergence", J.of Math. Anal. and Appl. vol. 76, pp. 571-599, 1980.

[15] Pu-Gui Shi, "A new definition of fuzzy compactness", Fuzzy sets and Systems vol. 158, pp. 14861495, 2007.

[16] R.Srivastava, S.N.Laland and A.K.Srivastava, "Fuzzy Hausdorff topological spaces", J. Math. Anal. Appl. vol. 81, pp. 497-506, 1981.

[17] G.J.Wang, "A new fuzzy compactness defined by fuzzy nets", J. of Math. Anal. and its Appl. vol. 94, pp. 1-23, 1983.

[18] R.H.Warren, "Fuzzy topologies characterized by neighborhood systems", Rocky Mountain J. Math. vol. 9, pp. 761-764, 1979.

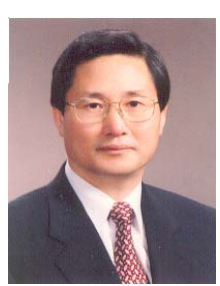

\section{Kyung Chan Min}

Professor of Yonsei University

Research Area : Fuzzy topology, General topology

E-mail : kcmin@yonsei.ac.kr

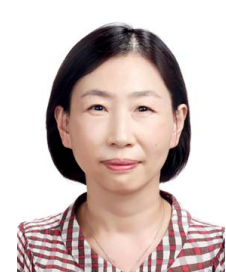

\section{Yoon Jin Lee}

Professor of Hankyong National University

Research Area : Topology

E-mail : yj1817@empas.com

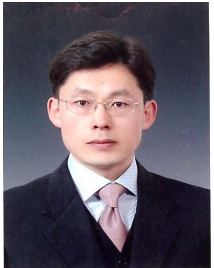

Jae Deuk Myung

Professor of Korea Aerospace University

Research Area : Topology

E-mail : mjd3954@hanmail.net 